\title{
A New Ranking Technique of Hexagonal Fuzzy Numbers
}

\author{
K. Dhurai ${ }^{1}$, A. Karpagam ${ }^{2}$ \\ ${ }^{1,2}$ Assistant Professor, Department of Mathematics, Valliammai Engineering College, Kattankulathur- 603203
}

\begin{abstract}
In this paper, we are presenting a new ranking technique with membership function for solving transportation problem, where fuzzy demand and supply are all in the form of Hexagonal fuzzy number. By defining a ranking to the Hexagonal fuzzy numbers, it is possible to compare them and using this we convert the fuzzy valued transportation problem to a crisp valued transportation problem, which then can be solved using the matrix minima method.
\end{abstract}

Keywords: Hexagonal fuzzy number, Ranking Technique, Membership function, Transportation Problem

\section{Introduction}

The transportation problem is a special case of linear programming problem, which enable us to determine the optimum shipping patterns between origins and destinations. A fuzzy transportation problem is a transportation problem in which the transportation costs, supply and demand quantities are fuzzy quantities.

The objective of the fuzzy transportation problem is to determine the shipping schedule that minimizes the total fuzzy transportation cost, which satisfying fuzzy supply and demand limits.

In section 2 preliminaries and section 3 the new ranking function are discussed with numerical example and section 4 concludes the paper.

\section{Preliminaries}

\subsection{Definition}

The characteristic function $\mu_{\mathrm{A}}$ of a crisp set A C X assigns a value either 0 or 1 to each member in $X$. This function can be generalized to a function $\mu_{A}$ such that the value assigned to the element of the universal set $X$ fall within a specified range i.e. $\mu_{A}: X \rightarrow[0,1]$. The assigned value indicate the membership function and the set $\widetilde{A}=\left\{\left(\mathrm{x}, \mu_{\mathcal{A}}(x)\right) ; \mathrm{x} \in \mathrm{X}\right\}$ defined by $\mu_{\mathcal{A}}(x)$ for $\mathrm{x} \epsilon \mathrm{X}$ is called fuzzy set.

\subsection{Definition}

A fuzzy set $\mathrm{A}$ of the real line $\mathrm{R}$ with membership function $\mu_{\mathcal{A}}$ : $X \rightarrow[0,1]$ is called fuzzy number if

i) A must be normal and convex fuzzy set.

ii) The support of $\bar{A}$ must be bounded

iii) $\alpha_{A}$ must be closed interval for every $\alpha \in[0,1]$

\subsection{Definition}

A fuzzy number $\widetilde{A_{H}}$ is a hexagonal fuzzy number denoted by $\widetilde{A_{H}}=\left(a_{1}, a_{2}, a_{\mathrm{a}}, a_{4}, a_{5}, a_{6}\right)$ where $\left(a_{1}, a_{2}, a_{\mathrm{a}}, a_{4}, a_{5}, a_{6}\right)$ are real numbers and its membership function $\mu_{\mathcal{A}_{H}}(x)$ is given below.

$$
\begin{aligned}
& \int_{1}^{0}\left(\frac{x-a_{1}}{a_{a}-a_{1}}\right) a_{1} \leq x \leq \frac{a_{1}+a_{a}}{2} \\
& \mu_{A_{H}}(x)=\left\{\begin{array}{c}
\frac{1}{2}+\frac{1}{2}\left(\frac{2 x-a_{1}-a_{a}}{a_{3}-a_{1}}\right) \frac{a_{1}+a_{a}}{2} \leq x \leq a_{a} \\
1 \quad a_{a} \leq x \leq a_{4} \\
1-\left(\frac{x-a_{4}}{a_{6}-a_{4}}\right) a_{4} \leq x \leq \frac{a_{4}+a_{6}}{2}
\end{array}\right. \\
& \left(\frac{x-a_{6}}{a_{4}-a_{6}}\right) \frac{a_{4}+a_{6}}{2} \leq x \leq a_{6} \\
& 0 \\
& x>a_{6}
\end{aligned}
$$

\subsection{Definition}

An effective approach for ordering the elements of $F(R)$ is also to define a ranking function $\Re: F(R) \rightarrow R$ which maps each fuzzy number into the real line, where a natural order exists. We define orders on $\mathrm{F}(\mathrm{R})$ by:

$\tilde{a} \geq$ bif and only if $\mathrm{R}(\tilde{a}) \geq \mathrm{R}(\tilde{b})$

$\tilde{a}>$ bif and only if $\mathrm{R}(\tilde{a}) \geq \mathrm{R}(\tilde{b})$

$\tilde{a}=\tilde{b} \quad$ if and only if $\mathrm{R}(\tilde{a})=\mathrm{R}(\tilde{b})$

\section{New Ranking Function}

\subsection{Alpha Cut}

The classical set $\boldsymbol{A}_{\bar{\alpha}}$ called alpha cut set is the set of elements whose degree of membership is the set of elements whose degree of membership in $\widetilde{A_{H}}=\left(a_{1}, a_{2}, a_{a}, a_{4}, a_{5}, a_{6}\right)$ is no less than, $\alpha$ it is defined as

$$
\begin{aligned}
& A_{\alpha}=\left\{\mathrm{x} \in \mathrm{X} / \mu_{A_{H}}(x) \geq \alpha\right\} \\
& \quad\left\{\begin{array}{l}
{\left[D_{1}(\alpha), D_{2}(\alpha)\right] \text { for } \alpha \in[0,0.5)} \\
{\left[S_{1}(\alpha), S_{2}(\alpha)\right] \text { for } \alpha \in[0,5,1]}
\end{array}\right. \\
& =\left\{\begin{array}{l}
D_{1}(\alpha)+S_{1}(\alpha) \text { for } \alpha \in[0,1] \\
D_{2}(\alpha)+S_{2}(\alpha) \text { for } \alpha \in[0,1]
\end{array}\right. \\
& =\left[\begin{array}{l}
\left.a_{1}+\alpha\left(a_{3}-a_{1}\right)+a_{6}+\alpha\left(a_{4}-a_{6}\right)\right] \text { for } \alpha \in[0,1]
\end{array}\right.
\end{aligned}
$$




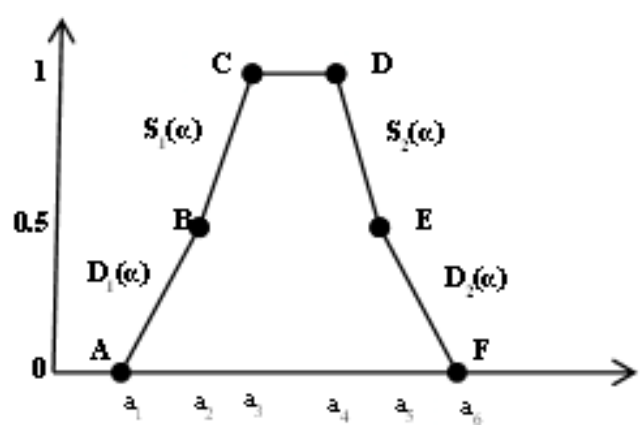

It provides results, $A$ is a fuzzy number then the ranking is defined by $R\left(\widetilde{A_{H}}\right)=\int_{0}^{1} 2(0.5)\left(A_{h \alpha}{ }^{L}, A_{h \alpha}{ }^{U}\right)$ d $\alpha$ where $\left(A_{h a}{ }^{\mathrm{L}}, A_{\mathrm{ha}}{ }^{\mathrm{U}}\right)$ is the $\alpha$ level cut of the fuzzy number $\widetilde{A_{H^{*}}}$ $R\left(\widetilde{A_{H}}\right)=\int_{0}^{1} 2(0.5)\left[a_{1}+\alpha\left(a_{a}-a_{1}\right)+a_{6}+\alpha\left(a_{4}-a_{6}\right)\right] d \alpha$

\subsection{Numerical Example}

A company has four warehouses $S L_{1}, S L_{2}, S L_{a}$ and $S L_{4}$. It is required to deliver a product from these warehouses to three customers $S G_{1}, S G_{2}$ and $S G_{\mathrm{g}}$. The warehouses have the following amounts in stock:

\begin{tabular}{|c|c|c|c|c|}
\hline Warehouse & $S L_{1}$ & $S L_{2}$ & $S L_{a}$ & $S L_{4}$ \\
\hline $\begin{array}{c}\text { Number.of.Un } \\
\text { its }\end{array}$ & $(4,5,6,7,8,9)$ & $(2,6,10,14,18$ & $(1,4,7,10,13$ & $(2,4,6,8,10,12$ \\
\hline
\end{tabular}

and the customers' requirements are

\begin{tabular}{|l|c|c|c|}
\hline Customers & $S G_{1}$ & $S G_{2}$ & $S G_{\mathrm{a}}$ \\
\hline Number.of. & $(10,11,12$, & $(5,7,9,11$, & $(6,8,10,12$, \\
Units & $13,14,15,16)$ & $13,15)$ & $14,16)$ \\
\hline
\end{tabular}

The table shows the costs of transporting one unit from warehouse to the customer.

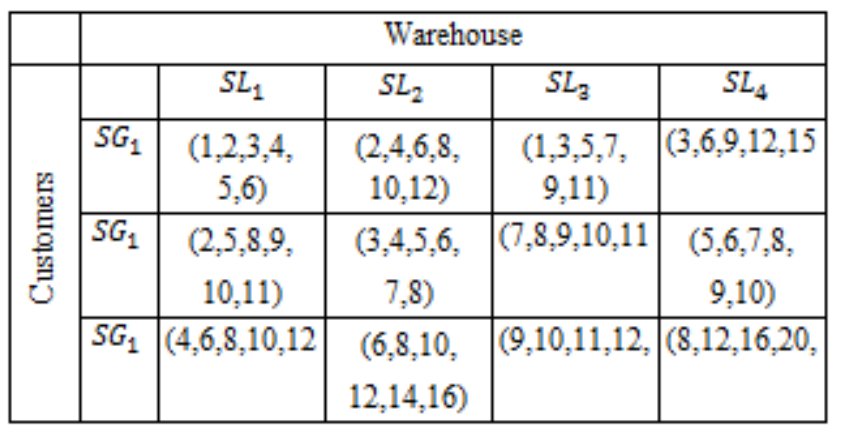

Solution: The fuzzy transportation problem can be formulated in the following mathematical programming from

Minimize $=$

$\Re(1,2,3,4,5,6)+\Re(2,4,6,8,10,12)+\Re(1,3,5,7,9,11)+\Re(3,6$, $9,12,15,18)+\Re(2,5,8,9,10,11)+\Re(3,4,5,6,7,8)+\Re(7,8,9,10$ $, 11,12)+\Re(5,6,7,8,9,10)+\Re(4,6,8,10,12,14)$ $+\Re(6,8,10,12,14,16)+\Re(9,10,11,12,13,14)+\Re(8,12,16,20$, 24,28)

$R\left(\widetilde{A_{H}}\right)=\int_{0}^{1} 2(0.5)\left[a_{1}+\alpha\left(a_{3}-a_{1}\right)+a_{6}+\alpha\left(a_{4}-a_{6}\right)\right] d \alpha$
$\Re(1,2,3,4,5,6)=\int_{0}^{1} 2(0.5)[1+\alpha(3-1)+6+\alpha(4-6)] \mathrm{d} \alpha=7$

Similarly

$\Re(2,4,6,8,10,12)=14 x$ R $(1,3,5,7,9,11)=12_{*}^{*}$

$\Re(3,6,9,12,15,18)=21 ; \Re(2,5,8,9,10,11)=13$;

$\Re(3,4,5,6,7,8)=11 ; \Re(7,8,9,10,11,12)=19$;

$\Re(5,6,7,8,9,10)=15 ; \Re(4,6,8,10,12,14)=18$;

$\Re(6,8,10,12,14,16)=22 ; \Re(9,10,11,12,13,14)=23$;

$\Re(8,12,16,20,24,28)=36 ; \Re(4,5,6,7,8,9)=13$;

$\Re(2,6,10,14,18,22)=24 ; \Re(1,4,7,10,13,16)=17$.

$\Re(2,4,6,8,10,12)=14 ; \Re(10,11,12,13,14,15,16)=26_{s}$

$\Re(5,7,9,11,13,15)=20 ; \Re(6,8,10,12,14,16)=22$.

Applying ranking formula, we get

\begin{tabular}{|c|c|c|c|c|c|}
\hline & $S L_{1}$ & $S L_{2}$ & $S L_{3}$ & $S L_{4}$ & Supply \\
\hline$S G_{1}$ & \begin{tabular}{|l|l|}
13 & 7 \\
\end{tabular} & 14 & 1312 & 21 & 26 \\
\hline$S G_{2}$ & 13 & $20 \mid 11$ & 19 & 15 & 20 \\
\hline$S G_{3}$ & 18 & $4 \sqrt{22}$ & $4 \sqrt{23}$ & \begin{tabular}{|l|l|}
14 & 36 \\
\end{tabular} & 22 \\
\hline Demand & 13 & 24 & 17 & 14 & \\
\hline
\end{tabular}

Therefore the minimum total cost is 1151 .

\section{Conclusion}

In this paper a new ranking technique obtained an optimal solution for a fuzzy transportation problem using Hexagonal fuzzy number. This method is very easily approach comparing to other method to solve fuzzy problem.

\section{References}

[1] Amit kumar, Jagdeep kaur and pushpinder sign, "Fuzzy optimal solution of fully fuzzy linear programming problems with inequality constraints," International Journal of mathematical and computer science, pp. 37 40, 2010.

[2] R.E Bellman and L.A Zadeh ,"Decision making in a fuzzy environment," Management sciences, vol.17, pp. 141-164, 1970.

[3] S.Chanas and D.Kuchta, "A concept of optimal solution of the transportation with fuzzy cost coefficient," fuzzy sets and systems, 82 (9) pp. 229-305, 1996.

[4] S.Chandrasekaran, G.Kokila and Junu saju, "Ranking of heptagon number using zero suffix Method," International Journal of Science and Research, vol.4, pp.2256-2257, 2015.

[5] S.Chandrasekaran, G.Kokila and Junu saju, " A new approach to solve fuzzy travelling salesman problems by using ranking functions," International Journal of Science and Research, vol.4, pp.2258-2259, 2015.

[6] K.Dhurai and A.Karpagam, " A new membership function on hexagonal fuzzy number," International Journal of Science and Research, vol.5, pp.1129-1131, 2016.

[7] Lu, Shiang.Tai and Chiang, Kao, "Solving fuzzy transportation problem based on extension principle," European Journal of Operational Research, 153, pp.661674, 2004.

Volume 5 Issue 6, June 2016 www.ijsr.net 
[8] P.Pandian and G.Natarajan,, A new algorithm for finding a fuzzy optimal solution for fuzzy transpotation problem," Applied Mathematical Sciences, vol.4, pp.7990, 2010.

[9] W.Ritha and J.Vinoths and J.Merline, "Multi-objective two stage fuzzy transportation problem, Journal of Physical science, vol.13, pp.107-120, 2009.

[10] P.Rajarajeswari, A.Sahaya Sudha, and R.Karthika, “ A New Operation on Hexagonal Fuzzy Number," International Journal of fuzzy Logic Systems, vol.3, No.3, pp-15-26, 2013.

[11] S. Rezvani, "Multiplication operation on Trapezoidal Fuzzy numbers," Journal of Physical sciences, vol. no. 15, pp.17-26, 2011.

[12] L.A.Zadeh, "Fuzzy Set," Information and Control, No.8, pp-338-353, 1965.

[13]H.J.Zimmermann, "Fuzzy Set theory and its applications," third edition, Klue Academic publishers, Bosten Massachusetts 1996.

Volume 5 Issue 6, June 2016 www.jijsr.net 